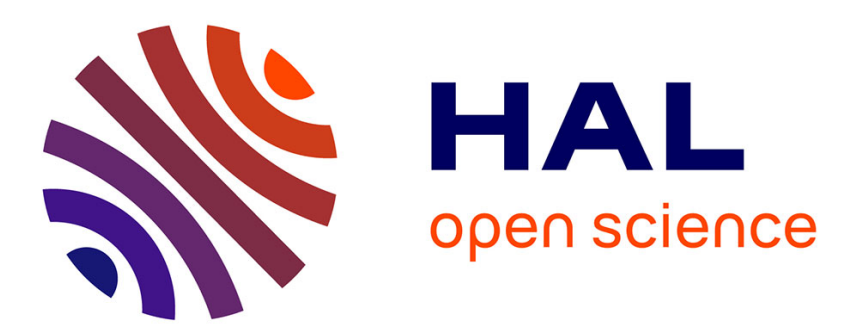

\title{
On The Relationship Between Differential Algebra and Tropical Differential Algebraic Geometry
}

François Boulier, Sebastian Falkensteiner, Marc Paul Noordman, Omar Leon

Sanchez

\section{- To cite this version:}

François Boulier, Sebastian Falkensteiner, Marc Paul Noordman, Omar Leon Sanchez. On The Relationship Between Differential Algebra and Tropical Differential Algebraic Geometry. Computer Algebra in Scientific Computing, Sep 2021, Sochi, Russia. pp.62-77. hal-03225922v2

HAL Id: hal-03225922

https://hal.science/hal-03225922v2

Submitted on 3 Jul 2021

HAL is a multi-disciplinary open access archive for the deposit and dissemination of scientific research documents, whether they are published or not. The documents may come from teaching and research institutions in France or abroad, or from public or private research centers.
L'archive ouverte pluridisciplinaire HAL, est destinée au dépôt et à la diffusion de documents scientifiques de niveau recherche, publiés ou non, émanant des établissements d'enseignement et de recherche français ou étrangers, des laboratoires publics ou privés. 


\title{
On The Relationship Between Differential Algebra and Tropical Differential Algebraic Geometry
}

\author{
François Boulier ${ }^{1}$, Sebastian Falkensteiner ${ }^{2}$, Marc Paul Noordman ${ }^{3}$, and Omar \\ León Sánchez ${ }^{4}$ \\ 1 Univ. Lille, CNRS, Centrale Lille, Inria, UMR 9189 - CRIStAL - Centre de \\ Recherche en Informatique Signal et Automatique de Lille, F-59000 Lille, France. \\ https://pro.univ-lille.fr/francois-boulier \\ 2 Research Institute for Symbolic Computation (RISC), Johannes Kepler University \\ Linz, Austria. https://risc.jku.at/m/sebastian-falkensteiner \\ 3 Bernoulli Institute, University of Groningen, The Netherlands. \\ https://www.rug.nl/staff/m.p.noordman \\ 4 University of Manchester, Department of Mathematics, United Kingdom. \\ omar.sanchez@manchester.ac.uk
}

\begin{abstract}
This paper presents the relationship between differential algebra and tropical differential algebraic geometry, mostly focusing on the existence problem of formal power series solutions for systems of polynomial ODE and PDE. Moreover, it improves an approximation theorem involved in the proof of the fundamental theorem of tropical differential algebraic geometry which permits to improve this latter by dropping the base field uncountability hypothesis used in the original version.
\end{abstract}

\section{Introduction}

Differential algebra is an algebraic theory for systems of ordinary or partial polynomial differential equations. It was founded by Ritt in the first half of the former century $[13,14]$ and developed by Kolchin [10]. Tropical differential algebraic geometry is a much more recent theory, founded by Grigoriev [8] aiming at applying the concepts of tropical algebra (aka min-plus algebra) to the study of formal power series solutions of systems of ODE. Tropical differential algebra obtained an important impulse by the proof of the fundamental theorem of tropical differential algebraic geometry [1] which was recently extended to the partial case in [7]. The common topic of both theories is the existence problem of formal power series solutions of polynomial differential equations on which an important paper [6] by Denef and Lipshitz was published in 1984.

In both [1] and [7], the fundamental theorem applies to a polynomial differential system $\Sigma$ with coefficients in formal power series rings $\mathscr{F}[[x]]$ (ordinary case) or $\mathscr{F}\left[\left[x_{1}, \ldots, x_{m}\right]\right]$ (partial case) where $\mathscr{F}$ is a characteristic zero differential field of constants which is both algebraically closed and uncountable. In this paper, we prove that the uncountability hypothesis can be dropped. Indeed, we prove 
that the fundamental theorem holds provided that $\mathscr{F}$ is algebraically closed and has countable ${ }^{5}$ transcendence degree over some field of definition $\mathscr{F}_{0}$ of $\Sigma$. This improvement of the fundamental theorem is achieved by generalizing the proof of a key proposition, which is an approximation theorem. This generalization is achieved in our Theorem 1, which is the main result of our paper. The new versions of the fundamental theorem, which follow, are stated in Theorem 2 and Theorem 3.

For the sake of simplicity, the introductory part of our paper focuses on the ordinary case. For completeness, the partial case is covered as well in the more technical sections. The paper is structured as follows. We recall in Section 2 the basic ideas underlying formal power series solutions of ODE and point out issues and known results, from the differential algebra literature. We state and explain the fundamental theorem of tropical differential algebra in Section 3. We provide our new approximation theorem in Section 5 (covering the partial differential case) and show how it is obtained by adapting the corresponding proposition given in [7]. The new version of the fundamental theorem, in the ordinary case, is provided in Section 6. In the final Section 7, we give an overview on the generalizations to the partial case, including the partial version of the fundamental theorem.

\section{Formal Power Series Solutions of ODE}

Let us start with a single autonomous ODE (i.e. an ODE the coefficients of which do not depend of the independent variable $x$ ) in a single differential indeterminate $y$ (standing for the unknown function $y(x)$ ):

$$
\dot{y}^{2}+8 y^{3}-1=0 \text {. }
$$

Differentiate it many different times.

$$
\begin{gathered}
2 \dot{y} \ddot{y}+24 y^{2} \dot{y}, \\
2 \dot{y} y^{(3)}+2 \ddot{y}^{2}+24 y^{2} \ddot{y}+48 y \dot{y}^{2},
\end{gathered}
$$

Rename each derivative $y^{(k)}$ as $v_{k}$. Solve the obtained polynomial system (observe there are infinitely many solutions). The result is a truncated arc $\underline{v}$

$$
\left(v_{0}, v_{1}, v_{2}, v_{3}, v_{4}, v_{5}, v_{6}, v_{7}, \ldots\right)=(0,1,0,0,-24,0,0,2880, \ldots) .
$$

Substitute the arc in the generic formula

$$
\Psi(\underline{v})=\sum \frac{v_{i}}{i !} x^{i} .
$$

One obtains a formal power series solution centered at the origin. Since the ODE is autonomous, the same arc, substituted in the following generic formula

$$
\Psi_{\alpha}(\underline{v})=\sum \frac{v_{i}}{i !}(x-\alpha)^{i}
$$

\footnotetext{
${ }^{5}$ In this paper, "countable" stands for "countably infinite".
} 
provides a formal power series solution centered at any expansion point $x=\alpha$.

If the ODE is not autonomous, the arc depends on the expansion point. The process is thus a variant. Consider some non-autonomous ODE

$$
x \dot{y}^{2}+8 x y^{3}-1=0 .
$$

Differentiate the ODE many different times.

$$
2 x \dot{y} \ddot{y}+\dot{y}^{2}+24 x y^{2} \dot{y}+8 y^{3},
$$

Then fix an expansion point $\alpha$ and evaluate the independent variable at $x=\alpha$. Solve the obtained polynomial system. The result is a truncated arc. Substitute it in (1) (for the chosen value of $\alpha$ ). One gets a formal power series solution centered at $x=\alpha$.

In the above processes, the only issue lies in the polynomial solving step. Indeed, each differentiated equation introduces a new leading derivative. These leading derivatives admit as leading coefficients the initial or the separant of the ODE. If these two polynomials do not vanish at the expansion point and the already secured coordinates of the truncated arc (the initial values, somehow, of the initial value problem), then the formal power series solution exists, is unique and straightforward to compute up to any term. However, if these polynomials vanish, the formal power series solution may fail to exist or be unique.

A device borrowed from [6, page 236] illustrates the issue. It shows how to build an ODE $p$ with coefficients in $\mathbb{Q}[x]$ from a polynomial $f(z)$ in $\mathbb{Q}[z]$. The ODE admits a formal power series solution centered at the origin if and only if the polynomial $f(z)$ has no positive integer solution. In the ordinary case, this device permits to build interesting examples. The approach generalizes to the partial case. It permits to relate the existence problem of formal power series solutions centered at the origin for PDE systems to Hilbert's Tenth Problem and Matiiassevich undecidability result [6, Theorem 4.11]. For more details see $[3$, Sect 1.6].

It is interesting also to observe that any non-autonomous ODE can be viewed as an autonomous one by performing a change of independent variable and introducing an extra ODE. Indeed, call $\xi$ the new independent variable. View the former independent variable $x$ as a new differential indeterminate (i.e. as an unknown function $x(\xi)$ ) and introduce the extra ODE $\dot{x}=1$. This reduction method only applies to ODE with polynomial coefficients in $x$. However, if $x=\alpha$ was a problematic expansion point before the reduction then $x(0)=\alpha$ becomes a problematic initial value (hence arc coordinate) after reduction. For more details see [3, Sect 1.4.2].

In his books [13,14], Ritt implicitly considers autonomous systems (the "autonomous" qualifier does not belong to differential algebra) and we may assume he had in mind the above reduction trick. Though Taylor expansions of solutions are discussed at different places (mostly in a chapter dedicated to PDE), Ritt does not explicitly address the existence problem of formal power series 
solutions. However, he pioneered differential elimination methods by means of his theory of characteristic sets (which was much developed afterwards, leading to the theories of regular chains and differential regular chains). This elimination theory solves in particular the following decision problem: given any finite system $\Sigma$ of ordinary or partial differential polynomial, does $1 \in[\Sigma]$ where $[\Sigma]$ denotes the differential ideal generated by $\Sigma$ ? This problem is equivalent to the following one, which is thus seen to be decidable: does there exist initial values for which $\Sigma$ has formal power series solutions ${ }^{6}$ ?

In the case of systems of non-autonomous ODE, thanks to the reduction method to the autonomous case, we can then conclude that the following problem is decidable: given any system $\Sigma$, do expansion point and initial values exist for which $\Sigma$ has formal power series solutions?

\section{The Fundamental Theorem of Tropical Differential Algebraic Geometry}

In the tropical differential case, the systems under consideration belong to some differential polynomial ring $\mathscr{F}[[x]]\left\{y_{1}, \ldots, y_{n}\right\}$ where $\mathscr{F}$ is a characteristic zero field of constants. Differential polynomials have formal power series coefficients. Thus the reduction trick to the autonomous case does not apply and formal power series solutions are only sought at a fixed expansion point: the origin. More precisely, formal power series solutions are sought in the coefficient ring $\mathscr{F}[[x]]$ of the equations.

The existence problem of such formal power series solutions is much more difficult. An important related paper is [6]. Indeed, [6, Theorem 3.1] claims that, in the case of systems with coefficients in $\mathbb{Q}[x]$, the existence problem of formal power series solutions (with coefficients in $\mathbb{C}, \mathbb{R}$ or $\mathbb{Q}_{p}$ ) is decidable. It is however important to note that, in the same setting, the existence problem of nonzero formal power series solutions is undecidable. See [6, Proposition 3.3] which refers to $[16]$.

In this context, the fundamental theorem of tropical differential geometry does not solve any problem left open in [6]. It only states the following equivalence

$$
\operatorname{supp}(\operatorname{sol}(\Sigma))=\operatorname{sol}(\operatorname{trop}(\Sigma))
$$

where $\Sigma$ is a differential ideal and the base field $\mathscr{F}$ is both algebraically closed and uncountable (we relinquish this last condition in this paper).

\footnotetext{
${ }^{6}$ Indeed, the characteristic sets or regular differential chains computed by differential elimination methods can be viewed as differential systems sufficiently simplified to generalize, for systems of differential equations, the basic methods sketched at the top of the section for computing formal power series solutions.
} 
Before entering sophisticated issues, let us clarify the notations used in (2). The $\operatorname{support}^{7} \operatorname{supp}(\varphi)$ of the formal power series (3) is the set $\left\{i \in \mathbb{N} \mid a_{i} \neq 0\right\}$.

$$
\varphi=\sum a_{i} x^{i}
$$

Since $\Sigma$ depends on $n$ differential indeterminates $y_{1}, \ldots, y_{n}$, its formal power series solutions actually are tuples of $n$ formal power series. One then extends the above definition to tuples of formal power series: the support of a tuple

$$
\varphi=\left(\varphi_{1}, \ldots, \varphi_{n}\right)
$$

is defined as the tuple $\operatorname{supp}(\varphi)=\left(\operatorname{supp}\left(\varphi_{1}\right), \ldots, \operatorname{supp}\left(\varphi_{n}\right)\right)$.

On the left hand side of $(2), \operatorname{sol}(\Sigma)$ denotes the set of formal power series solutions of $\Sigma$ with coefficients in $\mathscr{F}$. Hence, the left hand side of (2) is a set of tuples of the supports of all the formal power series solutions of $\Sigma$.

Let us address now the right hand side of (2). The valuation of a formal power series (3) is defined as $\infty$ if $\varphi=0$ and as the smallest $i \in \mathbb{N}$ such that $a_{i} \neq 0$ otherwise.

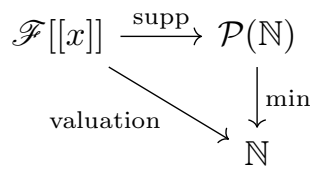

Let us now define the tropicalization of the differential monomial (the coefficient $c \in \mathscr{F}[[x]]$ and the term $t$ is a power product of derivatives $v_{1}, \ldots, v_{r}$ of the $n$ differential indeterminates $y_{1}, \ldots, y_{n}$ )

$$
m=c t=c v_{1}^{d_{1}} \cdots v_{r}^{d_{r}}
$$

at a tuple of supports

$$
S=\left(S_{1}, \ldots, S_{n}\right) .
$$

Consider any tuple of formal power series (4) whose support is $S$. Since $m$ is a monomial, the support of the formal power series $m(\varphi)$ is uniquely defined by $S$ : it does not depend on the actual coefficients of $\varphi$. We are led to the following definition ${ }^{8}$.

The tropicalization of a differential monomial $m$ at $S$ is defined as the valuation of $m(\varphi)$ where $\varphi$ is any tuple of formal power series whose support is $S$. The table below gives a few examples.

\begin{tabular}{|ccc|}
\hline \hline monomial $m$ & support $S$ & $\operatorname{trop}(m)$ at $S$ \\
\hline$x^{2} y$ & $\{0,1,2\}$ & 2 \\
$x^{2} y$ & $\{2\}$ & 4 \\
$\dot{y}^{3}$ & $\{0,3\}$ & 6 \\
$\ddot{y}^{3}$ & $\{0,1\}$ & $\infty$ \\
\hline \hline
\end{tabular}

\footnotetext{
${ }^{7}$ In $[1,8]$, the notation $\operatorname{trop}(\varphi)$ is used instead of $\operatorname{supp}(\varphi)$ but may be misleading in some cases.

8 This is not the definition given in $[1$, sect. 4$]$ but both definitions are equivalent.
} 
Let us now consider a nonzero differential polynomial, expanded as a sum of monomials of the form (5) with pairwise distinct terms:

$$
p=m_{1}+m_{2}+\cdots+m_{q} .
$$

The tropicalization of $p$ at $S$ is defined as

$$
\operatorname{trop}(p)=\min _{i=1}^{q} \operatorname{trop}\left(m_{i}\right) .
$$

The tropicalization of the zero polynomial is defined as $\infty$.

As an example, let us consider the differential polynomial

$$
p=\dot{y}^{2}-4 y
$$

whose solutions are $\varphi=0$ (support $S=\varnothing$ ) and $\varphi=(x+c)^{2}$ where $c$ is an arbitrary constant (supports $S=\{0,1,2\}$ and $\{2\}$ ). The first and second derivatives of $p$ are

$$
\begin{aligned}
& \dot{p}=2 \dot{y} \ddot{y}-4 \dot{y}, \\
& \ddot{p}=2 \dot{y} y^{(3)}+2 \ddot{y}^{2}-4 \ddot{y} .
\end{aligned}
$$

In the next table, all the considered supports are supports of solutions of the differential polynomials. In the last column, the list of the $\operatorname{trop}\left(m_{i}\right)$ is provided, rather than their minimum. The first row indicates that both monomials of $p$ vanish at $\varphi=0$. The second row indicates that the two monomials do not vanish but may possibly cancel each other at $\varphi=a_{2} x^{2}$, for some $a_{2} \neq 0$ (indeed, they vanish but only for $a_{2}=1$ ). The third row indicates that, among the three monomials of $\ddot{p}$, the first one vanishes at any $\varphi=a_{2} x^{2}$ while the two last ones may cancel each other for some $a_{2} \neq 0$.

\begin{tabular}{|ccc|}
\hline \hline polynomial & support $S$ & list trop $\left(m_{i}\right)$ at $S$ \\
\hline$p$ & $\varnothing$ & {$[\infty, \infty]$} \\
$p$ & $\{2\}$ & {$[2,2]$} \\
$\ddot{p}$ & $\{2\}$ & {$[\infty, 0,0]$} \\
\hline \hline
\end{tabular}

In the next table, the considered support $S=\{0,1\}$ is not the support of any solution of $p$, since $p$ has no solution of the form $\varphi=a_{0}+a_{1} x$ with $a_{0}, a_{1} \neq 0$. This fact is not observed on the first row, which considers $p$ itself. It is however observed on the second row, which considers the first derivative of $p$ : one of the two monomials vanishes while the second one evaluates to some nonzero formal power series.

\begin{tabular}{|ccc|}
\hline \hline polynomial & support $S$ & list trop $\left(m_{i}\right)$ at $S$ \\
\hline$p$ & $\{0,1\}$ & {$[0,0]$} \\
$\dot{p}$ & $\{0,1\}$ & {$[\infty, 0]$} \\
\hline \hline
\end{tabular}

The observed phenomena suggest the following definition, which permits to understand the right hand side of (2).

Let $p$ be a polynomial of the form (7). View $\operatorname{trop}(p)$ as a function of $n$ unknown supports. Then $\left(S_{1}, \ldots, S_{n}\right)$ is said to be a solution of $\operatorname{trop}(p)$ if either 
1. each $\operatorname{trop}\left(m_{i}\right)=\infty$ or

2. there exists $m_{i}, m_{j}(i \neq j)$ such that $\operatorname{trop}\left(m_{i}\right)=\operatorname{trop}\left(m_{j}\right)=\underset{k=1}{q}\left(\operatorname{trop}\left(m_{k}\right)\right)$.

Let us conclude this section by a few remarks. In the fundamental theorem of tropical differential algebraic geometry, the inclusion $\operatorname{supp}(\operatorname{sol}(\Sigma)) \subset$ $\operatorname{sol}(\operatorname{trop}(\Sigma))$ is easy. The difficult part is the converse inclusion. It requires $\Sigma$ to be a differential ideal because one may need to consider arbitrary high derivatives of the elements of $\Sigma$ in order to observe that a given support is not a solution. See the example above or even simpler, consider $p=\dot{y}-y$ and $S=\{0, \ldots, n\}$ with $n \in \mathbb{N}$ : it is necessary to differentiate $n$ times the differential polynomial $p$ in order to observe that it has no solution with support $S$. Moreover, the base field $\mathscr{F}$ is required to be algebraically closed because of the polynomial system solving step and the fact that solutions are sought in $\mathscr{F}[[x]]$.

Last, the proof of the converse inclusion relies on an approximation theorem. The two versions of this approximation theorem given in [1, Proposition 7.3] and [7, Proposition 6.3] assume $\mathscr{F}$ to be uncountable. Our new version (Theorem 1) relies on weaker hypotheses.

\section{Fields of Definition and Countability}

We are concerned with a differential ideal $\Sigma[10$, I, sect. 2] in a characteristic zero partial differential polynomial ring $\mathscr{F}\left[\left[x_{1}, \ldots, x_{m}\right]\right]\left\{y_{1}, \ldots, y_{n}\right\}$ where $\mathscr{F}$ is an algebraically closed field of constants, the $m$ derivation operators $\delta_{1}, \ldots, \delta_{m}$ act as $\partial / \partial x_{1}, \ldots, \partial / \partial x_{m}$ and $y_{1}, \ldots, y_{n}$ are $n$ differential indeterminates.

Thanks to the Ritt-Raudenbush Basis Theorem (see [4] for details), the differential ideal $\Sigma$ can be presented by finitely many differential polynomials $g_{1}, \ldots, g_{s} \in \mathscr{F}\left[\left[x_{1}, \ldots, x_{m}\right]\right]\left\{y_{1}, \ldots, y_{n}\right\}$ in the sense that the perfect $[10,0$, sect. 5] differential ideals $\{\Sigma\}$ and $\left\{g_{1}, \ldots, g_{s}\right\}$ are equal.

A field of definition ${ }^{9}$ of $\Sigma$ is any subfield $\mathscr{F}_{0} \subset \mathscr{F}$ such that there exist $g_{1}, \ldots, g_{s} \in \Sigma \cap \mathscr{F}_{0}\left[\left[x_{1}, \ldots, x_{m}\right]\right]\left\{y_{1}, \ldots, y_{n}\right\}$ with $\Sigma \subseteq\left\{g_{1}, \ldots, g_{s}\right\}$ (the perfect differential ideal generated by $\left.g_{1}, \ldots, g_{s}\right)$.

Proposition 1. Any differential ideal $\Sigma$ has a countable algebraically closed field of definition $\mathscr{F}_{0}$. Moreover, if $\mathscr{F}$ has countable transcendence degree over $\mathscr{F}_{0}$ then $\mathscr{F}$ also is countable.

Proof. Let $S$ be the family of the coefficients of the formal power series coefficients of any basis of $\Sigma$ which are transcendental over the field $\mathbb{Q}$ of the rational numbers. The family $S$ is countable. An algebraically closed field of definition $\mathscr{F}_{0}$ can be defined as the algebraic closure of $\mathbb{Q}(S)$.

Now, the field $\mathbb{Q}$ is countable. If $\mathscr{L}$ is a countable field and $S$ is a countable family of transcendental elements over $\mathscr{L}$ then $\mathscr{L}(S)$ is countable. Moreover, if $\mathscr{L}$ is countable then its algebraic closure is countable [9, Theorem 65].

The last statement of the proposition follows using the same arguments.

In the sequel, $\mathscr{F}_{0}$ denotes an algebraically closed field of definition of $\Sigma$.

\footnotetext{
9 This definition is adapted from [10, I, sect. 5].
} 


\section{The Approximation Theorem}

Denote by $\Theta$ the commutative semigroup of the derivative operators generated by the derivation operators i.e. $\Theta=\left\{\delta_{1}^{a_{1}} \cdots \delta_{m}^{a_{m}} \mid a_{1}, \ldots, a_{m} \in \mathbb{N}\right\}$.

Define a one-to-one correspondence between the set of all pairs $(i, \theta) \in$ $[1, n] \times \Theta$ and the set $\mathbb{N}$ of nonnegative integers. This correspondence permits us to enumerate all derivatives $\theta y_{i}$ of the differential indeterminates. Fix a correspondence which defines an orderly ranking (derivatives are enumerated by increasing order) [10, chap. I, sect. 8]. The derivatives of the $y$ are denoted $v_{0}, v_{1}, v_{2}, \ldots$

As in Section 4 , let $\Sigma$ be a differential ideal included in the perfect differential ideal $\left\{g_{1}, \ldots, g_{s}\right\}$ generated by $g_{1}, \ldots, g_{s} \in \Sigma$ with field of definition equal to $\mathscr{F}_{0}$. Define another one-to-one correspondence between the set of all pairs $(i, \theta) \in$ $[1, s] \times \Theta$ and $\mathbb{N}$. This correspondence permits us to enumerate all derivatives $\theta g_{i}$. Again, fix a correspondence which defines an orderly ranking on the derivatives of the $g$ (viewing them as $s$ differential indeterminates). The derivatives of the $g$, evaluated at $x_{1}=\cdots=x_{m}=0$, are denoted $f_{0}, f_{1}, f_{2}, \ldots$ The polynomials $f$ thus belong to $\mathscr{F}_{0}\left\{y_{1}, \ldots, y_{n}\right\}$.

Let $k$ be a positive integer. Denote

$$
\begin{aligned}
\Sigma_{k} & =\left\{f_{i} \mid 0 \leq i \leq k\right\}, \\
\Sigma_{\infty} & =\left\{f_{i} \mid i \in \mathbb{N}\right\} .
\end{aligned}
$$

Define $\kappa(k)=\kappa$ as the smallest integer such that $\Sigma_{k} \subset \mathscr{F}_{0}\left[v_{0}, \ldots, v_{\kappa}\right]$. The index $\kappa$ exists because the ranking is orderly. Define

$$
A_{k}=\left\{a \in \mathscr{F}_{0}^{\kappa+1} \mid f_{0}(a)=\cdots=f_{k}(a)=0\right\} .
$$

Let now $S$ be any subset of $\mathbb{N}$. Define $A_{k, S}$ as the set of zeros of $A_{k}$ which are compatible with $S$ :

$$
A_{k, S}=\left\{a \in A_{k} \mid a_{i} \neq 0 \text { if and only if } i \in S \cap[0, \kappa]\right\} .
$$

Indeed, thanks to the fixed one-to-one correspondence between the derivatives of the differential indeterminates and the set $\mathbb{N}$, any such set $S$ encodes a tuple of $n$ supports of formal power series. Given any field extension $\mathscr{E}$ of $\mathscr{F}_{0}$, define

$$
\begin{aligned}
A_{\infty}(\mathscr{E}) & =\left\{a \in \mathscr{E}^{\mathbb{N}} \mid f_{i}(a)=0 \text { for each } i \in \mathbb{N}\right\} \\
A_{\infty, S}(\mathscr{E}) & =\left\{a \in A_{\infty}(\mathscr{E}) \mid a_{i} \neq 0 \text { if and only if } i \in S\right\}
\end{aligned}
$$

The elements of $A_{\infty}(\mathscr{F})$ give exactly the formal power series solutions of $\Sigma$. The elements of $A_{\infty, S}(\mathscr{F})$ give the formal power series solutions whose supports are encoded by $S$.

Theorem 1. Assume $\mathscr{F}$ has countable transcendence degree over $\mathscr{F}_{0}$ and is algebraically closed. Let $S$ be any subset of $\mathbb{N}$. If $A_{k, S} \neq \varnothing$ for each $k \in \mathbb{N}$ then $A_{\infty, S}(\mathscr{F}) \neq \varnothing$. 
There are many proofs which have the following sketch in common:

1. one first proves that $\Sigma_{\infty}$ admits a solution compatible with $S$ in some (big) field extension $\mathscr{E}$ of $\mathscr{F}_{0}$. This solution is an arc $a=\left(a_{i}\right)$ with coordinates $a_{i} \in \mathscr{E}$ for $i \in \mathbb{N}$. With other words, $A_{\infty, S}(\mathscr{E}) \neq \varnothing$;

2. the arc $a$ can be mapped to another $\operatorname{arc} \phi(a)$ with coordinates in $\mathscr{F}$ which is also a solution of $\Sigma_{\infty}$ compatible with $S$. Thus $A_{\infty, S}(\mathscr{F}) \neq \varnothing$ and Theorem 1 is proved.

There are actually many different ways to prove Step 1 above. The next sections provide three different variants.

\subsection{Proof of Step 1 by Ultraproducts}

The idea of this proof is mostly due do Marc Paul Noordman. It is inspired by techniques used in [6]. A minimal introduction of ultraproducts for casual readers is provided in Section A.

Proof. Let $\mathscr{R}$ be the ring obtained by inversion of all derivatives with indices in $S$ and quotient by the ideal equal to the sum of the ideal generated by $\Sigma_{\infty}$ and the ideal generated by the derivatives with indices not in $S$, i.e.

$$
\mathscr{R}=\mathscr{F}_{0}\left[v_{i}, v_{j}^{-1} \mid i \in \mathbb{N}, j \in S\right] /\left(f_{i}, v_{j} \mid i \in \mathbb{N}, j \notin S\right) .
$$

By Lemma 1 (below), this ring is not the null ring. By Krull's Theorem, it contains a maximal ideal $\mathfrak{m}$. A suitable field extension $\mathscr{E}$ of $\mathscr{F}_{0}$ is given by $\mathscr{R} / \mathfrak{m}$. The coordinates of the arc $\left(a_{i}\right)$ are the images of the derivatives $v_{i}$ by the natural $\mathscr{F} 0$-algebra homomorphism $\mathscr{R} \rightarrow \mathscr{R} / \mathfrak{m}$.

Lemma 1. The ring $\mathscr{R}$ defined in (12) is not the null ring.

Proof. We prove the lemma by showing that $\Sigma_{\infty}$ admits a solution in some field $\mathscr{F}_{0}^{*}$ (which turns out to be an ultrafield - see Section A) and constructing a map $\mathscr{F}_{0}\left\{y_{1}, \ldots, y_{n}\right\} \rightarrow \mathscr{F}_{0}^{*}$ which factors as $\mathscr{F}_{0}\left\{y_{1}, \ldots, y_{n}\right\} \rightarrow \mathscr{R} \rightarrow \mathscr{F}_{0}^{*}$.

To each $k \in \mathbb{N}$ associate an element $a^{k} \in A_{k, S}$. We have

$$
a^{k}=\left(a_{0}^{k}, a_{1}^{k}, \ldots, a_{\kappa}^{k}\right) \in \mathscr{F}_{0}^{\kappa} .
$$

Fix any non principal ultrafilter $\mathscr{D}$ on $\mathbb{N}$ and consider the ultrafield $\mathscr{F}_{0}^{*}=$ $\left(\prod_{i \in \mathbb{N}} \mathscr{F}_{0}\right) / \mathscr{D}$. For each $i \in \mathbb{N}$ define $u_{i} \in \mathscr{F}_{0}^{*}$ by

$$
\begin{aligned}
u_{0} & =\left(a_{0}^{0}, a_{0}^{1}, a_{0}^{2}, \ldots, a_{0}^{k}, \ldots\right), \\
u_{1} & =\left(a_{1}^{0}, a_{1}^{1}, a_{1}^{2}, \ldots, a_{1}^{k}, \ldots\right), \\
& \vdots \\
u_{i} & =\left(a_{i}^{0}, a_{i}^{1}, a_{i}^{2}, \ldots, a_{i}^{k}, \ldots\right),
\end{aligned}
$$


On each column $k$ of the above "array", the elements $a_{i}^{k}$ such that $i>\kappa$ are not defined. Set them to zero. Observe that on each row, there are only finitely many such elements.

We have thus defined a map $v_{i} \mapsto u_{i}$.

Let now $i \in \mathbb{N}$ be the index of some polynomial $f_{i}=f$. Evaluate $f$ to an element of $\mathscr{F}_{0}^{*}$ by substituting $u_{j}$ to $v_{j}$ for each $j \in \mathbb{N}$. Ultrafield operations are performed componentwise and the zeros of the $f$ appear on the columns in the above array. Thus, $f$ evaluates to zero over the $k$ th coordinate of $\mathscr{F}_{0}^{*}$ for all sufficiently large values of $k$. This set of values of $k$ is cofinite and hence, $f$ evaluates to zero in $\mathscr{F}_{0}^{*}$.

Let now $i \in \mathbb{N}$ be the index of some derivative $v_{i}$. By definition of $A_{k, S}$, if $i \notin S$ then all the coordinates of $u_{i}$ are zero so that $u_{i}$ is zero in $\mathscr{F}_{0}^{*}$; if $i \in S$ then the coordinates $a_{i}^{k}$ of $u_{i}$ are nonzero for all sufficiently large values of $k$ and $u_{i}$ is nonzero in $\mathscr{F}_{0}^{*}$.

The mapping $v_{i} \mapsto u_{i}$ thus defines a zero of $\Sigma_{\infty}$ which is compatible with $S$ and with coordinates in $\mathscr{F}_{0}^{*}$.

\subsection{Proof of Step 1 by a Model Theoretic Argument}

The idea of this proof is due to Omar León Sánchez.

Proof. Define

$$
\Omega(v)=\left\{f_{i}=0 \mid i \in \mathbb{N}\right\} \cup\left\{v_{i}=0 \mid i \notin S\right\} \cup\left\{v_{i} \neq 0 \mid i \in S\right\} .
$$

For any subcollection $\Omega_{0}(v)$ of $\Omega(v)$, there is a large enough $k \in \mathbb{N}$ such that if $a \in A_{k, S}$ then $a$ is a solution of $\Omega_{0}(v)$. Hence the assumption that $A_{k, S} \neq \varnothing$, for all $k \in \mathbb{N}$, yields that $\Omega(v)$ is finitely satisfiable.

By the compactness theorem in first-order logic (see for instance [12, Chapter $3]$ ) applied in the context of fields, the fact that $\Omega(v)$ is finitely satisfiable implies that there is a field extension $\mathscr{E}$ of $\mathscr{F}_{0}$ and an arc $a=\left(a_{i}\right)$ with coordinates in $\mathscr{E}$ solving $\Omega(v)$.

Remark 1. We note that Theorem 1 should not be too surprising to a modeltheorist; as it can be seen as an application of general results on strongly minimal theories (for instance, the fact that in a strongly minimal theory there is a unique non-algebraic complete 1-type over any set of parameters). Here the theory in mind is algebraically closed fields and the slightly more general result is as follows: Let $x=\left(x_{i}\right)_{i \in I}$ be a tuple of variables and $L / K$ a field extension of transcendence degree at least $|I|$ with $L$ algebraically closed. Suppose $T(x)$ is a collection of polynomial equations and in-equations over $K$. If $T(x)$ is finitely satisfiable, then there is a solution of $T(x)$ in $L$.

\subsection{Proof of Step 1 by Lang's Infinite Nullstellensatz}

The idea of this proof was suggested by an anonymous reviewer. 
Proof. Enlarge the set of derivatives $\left(v_{i}\right)$ with another infinite set of derivatives $\left(w_{i}\right)$ where $i \in \mathbb{N}$. Define

$$
\Omega_{\infty}=\Sigma_{\infty} \cup\left\{v_{i} \mid i \notin S\right\} \cup\left\{v_{i} w_{i}-1 \mid i \in S\right\} .
$$

Any solution of $\Omega_{\infty}$ provides a solution of $\Sigma_{\infty}$ which is compatible with $S$. The set of variables $v, w$ is indexed by $\mathbb{N}$. Let $\mathscr{E}$ be any uncountable field. Note that the ideal generated by $\Omega_{\infty}$ in the polynomial ring $\mathscr{E}[v, w]$ is proper; otherwise, 1 could be written as a linear combination (over $\mathscr{E}[v, w]$ ) of finitely many of the elements of $\Omega_{\infty}$, but this implies $A_{k, S}=\varnothing$ for some large enough $k \in \mathbb{N}$ (contradicting our hypothesis). Then, by [11, Theorem, conditions (ii) and S2], the system $\Omega_{\infty}$ has a solution in $\mathscr{E}$. Thus $A_{\infty, S}(\mathscr{E}) \neq \varnothing$.

\subsection{Proof of Step 2}

In Step 1, we have proved that there exists a field extension $\mathscr{E}$ of $\mathscr{F}_{0}$ such that $A_{\infty, S}(\mathscr{E}) \neq \varnothing$. Let us prove that $A_{\infty, S}(\mathscr{F}) \neq \varnothing$.

Proof. Consider some $a \in A_{\infty, S}(\mathscr{E})$. Let $J \subset \mathbb{N}$ be such that $\left(a_{j}\right)_{j \in J}$ is a transcendence basis of $\mathscr{F}_{0}(a)$ over $\mathscr{F}_{0}$. Denote $\mathscr{F}_{1}$ the algebraic closure of $\mathscr{F}_{0}\left(a_{j}\right)_{j \in J}$. Then the full arc $a$ has coordinates in $\mathscr{F}_{1}$. Since $\mathscr{F}$ has countable transcendence degree over $\mathscr{F}_{0}$ we have $\operatorname{trdeg}\left(\mathscr{F} / \mathscr{F}_{0}\right) \geq \operatorname{trdeg}\left(\mathscr{F}_{1} / \mathscr{F}_{0}\right)=|J|$. Moreover, since $\mathscr{F}$ is algebraically closed, there exists a $\mathscr{F}_{0}$-algebra homomorphism $\phi: \mathscr{F}_{1} \rightarrow \mathscr{F}$ such that $\phi(a)$ is a solution of $\Sigma_{\infty}$ compatible with $S$. Thus $A_{\infty, S}(\mathscr{F}) \neq \varnothing$.

\section{The New Version of the Fundamental Theorem}

For completeness, we provide the part of the proof of the fundamental theorem which makes use of our Theorem 1 . The proof is the same as that of [1, Theorem 8]. We start with an easy Lemma [1, Remark 4.1].

Lemma 2. Let $S=\left(S_{1}, \ldots, S_{n}\right)$ be a tuple of $n$ supports and $m=c v_{1}^{d_{1}} \cdots v_{r}^{d_{r}}$ be a monomial. Then $\operatorname{trop}(m)=0$ at $S$ if and only if the valuation of $c$ is zero and each factor $v^{d}=\left(y_{j}^{(k)}\right)^{d}$ of $m$ is such that $k \in S_{j}$.

Before stating the fundamental theorem, let us stress that the fields $\mathscr{F}_{0}$ and $\mathscr{F}$ mentioned in Theorem 2 can be assumed to be countable, by Proposition 1.

Theorem 2 (Fundamental Theorem for ODE).

Let $\Sigma$ be a differential ideal of $\mathscr{F}[[x]]\left\{y_{1}, \ldots, y_{n}\right\}$ where $\mathscr{F}$ is an algebraically closed field of constants and $\mathscr{F}_{0}$ be an algebraically closed field of definition of $\Sigma$. If $\mathscr{F}$ has countable transcendence degree over $\mathscr{F}_{0}$ then

$$
\operatorname{supp}(\operatorname{sol}(\Sigma))=\operatorname{sol}(\operatorname{trop}(\Sigma))
$$


Proof. Let us first address a few particular cases.

Case 1: there exists some nonzero $c \in \Sigma \cap \mathscr{F}[[x]]$. Differentiating $c$ sufficiently many times, we see that $1 \in \Sigma$. Then, on the one hand, $\operatorname{supp}(\operatorname{sol}(\Sigma))=\varnothing$. On the other hand, $\operatorname{sol}(\operatorname{trop}(1))=\varnothing$. Thus the theorem holds in Case 1 .

Case 2: $\Sigma=(0)$. Then, on the one hand $\operatorname{supp}(\operatorname{sol}(\Sigma))$ contains all supports. On the other hand, sol(trop $(0))$ contains all supports too. Thus the theorem holds in Case 2.

Let us now address the general case. The inclusion $\subset$ is easy. We prove the converse one. We assume that $S$ is not the support of any solution of $\Sigma$ and we show that $S$ is not a solution of $\operatorname{trop}(\Sigma)$.

For this, we are going to build a differential polynomial $h \in \Sigma$, expanding to a sum of monomials

$$
h=m_{1}+m_{2}+\cdots+m_{r},
$$

such that $\operatorname{trop}\left(m_{1}\right)=0$ and $\operatorname{trop}\left(m_{i}\right)>0$ for $2 \leq i \leq r$.

By the Ritt-Raudenbush Basis Theorem (see [4] for details), there exists a finite set $g_{1}, \ldots, g_{s}$ of differential polynomials of $\Sigma$ such that the solution set of $\Sigma$ is the solution set of the differential ideal $\left[g_{1}, \ldots, g_{s}\right]$ generated by the $g$.

From now on, we use the notations introduced in Section 5. Since $\left[g_{1}, \ldots, g_{s}\right]$ has no solution with support $S$ we have $A_{\infty, S}(\mathscr{F})=\varnothing$ whence, by Theorem 1, there exists some index $k$ such that $A_{k, S}=\varnothing$. Recall that $A_{k, S}$ is a subset of the algebraic variety of some polynomial system obtained by prolonging, and evaluating at $x=0$, the system of the $g$ up to some order and that the prolonged system belongs to some polynomial ring $\mathscr{F}_{0}\left[v_{1}, \ldots, v_{\kappa}\right]$.

Claim: there exists a differential polynomial

$$
\hat{h}=\hat{m}_{1}^{d}+\hat{m}_{2}+\cdots+\hat{m}_{\hat{r}}
$$

in the ideal $\left(f_{0}, \ldots, f_{k}\right)$ of the Noetherian polynomial ring $\mathscr{F}_{0}\left[v_{1}, \ldots, v_{\kappa}\right]$ such that $\operatorname{trop}\left(\hat{m}_{1}\right)=0$ and $\operatorname{trop}\left(\hat{m}_{i}\right)>0$ for $2 \leq i \leq \hat{r}$.

The ideal $\left(f_{0}, \ldots, f_{k}\right)$ has no solution compatible with $S$. This means that (the right hand side of the first line holds only for a non-empty support $S$ but the one of the second line holds in general):

$$
\begin{aligned}
{\left[f_{0}=\cdots=f_{k}=0 \text { and } v_{j}=0 \text { for all } v_{j} \text { s.t. } j \notin S\right] } & \Rightarrow v_{\ell}=0 \text { for some } \ell \in S \\
& \Rightarrow \underbrace{\prod_{\ell \in S, \ell \leq \kappa} v_{\ell}}_{\hat{m}_{1}}=0 .
\end{aligned}
$$

By Lemma 2 we have $\operatorname{trop}\left(\hat{m}_{1}\right)=0$ at $S$. By Hilbert's Nullstellensatz, we have

$$
\hat{m}_{1} \in \sqrt{\left(f_{0}, \ldots, f_{k},\left(v_{j}\right)_{j \notin S}\right)}
$$

Thus there exists a positive integer $d$ and monomials $\hat{m}_{2}, \ldots, \hat{m}_{\hat{r}}$ defining the polynomial $\hat{h}$ as in (15). We have $\hat{h} \in\left(f_{0}, \ldots, f_{k}\right)$ and for each $2 \leq i \leq \hat{r}$, 
there exists some $j \notin S$ for which $\operatorname{deg}\left(\hat{m}_{i}, v_{j}\right)>0$. By Lemma 2, we thus have $\operatorname{trop}\left(\hat{m}_{i}\right)>0$ at $S$ for each $2 \leq i \leq \hat{r}$. The claim is thus proved.

Now, since $\hat{h} \in\left(f_{0}, \ldots, f_{k}\right)$ we see that $\hat{h}$ can also be obtained by evaluating at $x=0$ some polynomial $h \in \Sigma$. Consider any monomial $m$ of $h$, of the form (5). If the evaluation at $x=0$ maps $m$ to zero then the valuation of the coefficient $c$ of $m$ is positive. In such a case, $\operatorname{trop}(m)>0$ by Lemma 2. If it maps $m$ to some nonzero monomial $\hat{m}$ then the valuation of $c$ is nonzero, both $m$ and $\hat{m}$ share the same term and $\operatorname{trop}(m)=\operatorname{trop}(\hat{m})$. Thus the polynomial $h$ has the form (14) and the theorem is proved.

\section{$7 \quad$ The Partial Differential Case}

In this section we give an overview on the generalization to the case of partial differential equations.

We seek for solutions of systems $\Sigma \subset \mathscr{F}\left[\left[x_{1}, \ldots, x_{m}\right]\right]\left\{y_{1}, \ldots, y_{m}\right\}$ in the ring of multivariate formal power series $\mathscr{F}\left[\left[x_{1}, \ldots, x_{m}\right]\right]$.

In this case, the algorithmic problems are even worse than in the ordinary case. According to [6, Theorem 4.11], there even cannot be an algorithm for deciding solvability of linear systems, a subclass of algebraic differential equations as we consider. Instead of actually computing the solutions of $\Sigma$, we again present an equivalent description of the solutions in the form of (2).

As in the ordinary case $(m=1)$, the support of a formal power series

$$
\varphi=\sum a_{I} x^{I}=\sum a_{\left(i_{1}, \ldots, i_{m}\right)} x_{1}^{i_{1}} \cdots x_{m}^{i_{m}}
$$

is the set $\left\{I \in \mathbb{N}^{m} \mid a_{I} \neq 0\right\}$. Hence, the left hand side of (2) is defined also for $m>1$.

For the tropicalization of $\Sigma$, the generalization cannot be done straightforward, since there is no well-defined minimum of elements in $\mathbb{N}^{m}$. In [7] is used instead a very specific partial order induced by vertex sets, which we briefly describe here.

Let $X \subset \mathbb{N}^{m}$. The Newton polytope $\mathcal{N}(X) \subseteq \mathbb{R}_{\geq 0}^{m}$ of $X$ is defined as the convex hull of $X+\mathbb{N}^{m}=\left\{x+n \mid x \in X, n \in \mathbb{N}^{m}\right\}$. Moreover, $x \in X$ is called a vertex if $x \notin \mathcal{N}(X \backslash\{x\})$, and vert is the set of vertices of $X$. It follows that $\operatorname{vert}(X)$ is the minimal set in $\mathbb{N}^{m}$ (with respect to the relation " $\subset$ ") generating $\mathcal{N}(X)$. Let us denote all vertex sets as $\mathbb{T}_{m}=\left\{\right.$ vert $\left.\mid X \subset \mathbb{N}^{m}\right\}$. Then, the composition of taking the support and then its vertex set of the formal power series defines a non-degenerate valuation such that some ideas of [1] can be recovered.

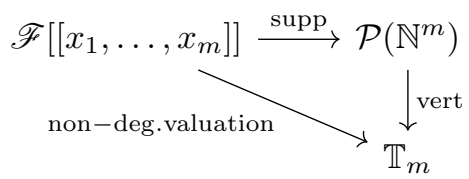

The tropicalization of a differential monomial $m=c v_{1}^{d_{1}} \cdots v_{r}^{d_{r}}$ at a tuple of supports $S=\left(S_{1}, \ldots, S_{n}\right) \subset\left(\mathbb{N}^{m}\right)^{n}$ is defined as the non-degenerate valuation 
of $m(\varphi)$ from above with $\operatorname{supp}(\varphi)=S$. Let us illustrate this.

\begin{tabular}{|ccc|}
\hline \hline monomial $m$ & support $S$ & $\operatorname{trop}(m)$ at $S$ \\
\hline$x_{1} x_{2} y$ & $\{(1,0),(0,1)\}$ & $\{(2,0),(0,2)\}$ \\
$\left(\frac{\partial y}{\partial x_{1}}\right)^{2}$ & $\{(2,0),(0,2)\}$ & $\{(2,0)\}$ \\
$\left(\frac{\partial^{2} y}{\partial x_{1} \partial x_{2}}\right)^{2}$ & $\{(2,0),(0,2)\}$ & $\varnothing$ \\
\hline \hline
\end{tabular}

The tropicalization of a differential polynomial $p$ of the form (5) at $S$ is defined as

$$
\operatorname{trop}(p)=\operatorname{vert}\left(\bigcup_{i=1}^{q} \operatorname{trop}\left(m_{i}\right)\right) .
$$

Let us consider the polynomial

$$
p=\frac{\partial y}{\partial x_{1}} \cdot \frac{\partial y}{\partial x_{2}}+\left(-x_{1}^{2}+x_{2}^{2}\right) \frac{\partial^{2} y}{\partial x_{1} \partial x_{2}} .
$$

\begin{tabular}{|ccc|}
\hline \hline polynomial & support $S$ & list trop $\left(m_{i}\right)$ at $S$ \\
\hline$p$ & $\{(2,0),(0,2)\}$ & {$[\{(1,1)\}, \varnothing]$} \\
$p$ & $\{(2,0),(1,1),(0,2)\}$ & {$[\{(2,0),(1,1),(0,2)\},\{(2,0),(0,2)\}]$} \\
\hline \hline
\end{tabular}

Considering $\operatorname{trop}(p)$ as a function of $n$ unknown supports, $\left(S_{1}, \ldots, S_{n}\right)$ is said to be a solution of $\operatorname{trop}(p)$ if for every vertex $J \in \operatorname{trop}(p)$ there exists $m_{i}, m_{j}$ $(i \neq j)$ such that $J \in \operatorname{trop}\left(m_{i}\right) \cap \operatorname{trop}\left(m_{j}\right)$. In the example above, we see that there cannot be a solution of $p$ with support equals $\{(2,0),(0,2)\}$, but $\varphi=$ $x_{1}^{2}+2 x_{1} x_{2}-x_{2}^{2}$ is indeed a solution.

For more illustrations of the tropicalization of (partial) differential polynomials, see [5].

As in the ordinary case, the inclusion $\operatorname{supp}(\operatorname{sol}(\Sigma)) \subset \operatorname{sol}(\operatorname{trop}(\Sigma))$ in the fundamental theorem is relatively easy [7, Proposition 5.7]. The converse inclusion can be shown exactly as in [7, Section 6], except that we replace Proposition 6.3 by the Approximation Theorem 1.

For consistency let us recall the main result here.

Theorem 3 (Fundamental Theorem for PDE). Let $\Sigma$ be a differential ideal of $\mathscr{F}\left[\left[x_{1}, \ldots, x_{m}\right]\right]\left\{y_{1}, \ldots, y_{n}\right\}$ where $\mathscr{F}$ is an algebraically closed field of constants and $\mathscr{F}_{0}$ be an algebraically closed field of definition of $\Sigma$. If $\mathscr{F}$ has countable transcendence degree over $\mathscr{F}_{0}$ then

$$
\operatorname{supp}(\operatorname{sol}(\Sigma))=\operatorname{sol}(\operatorname{trop}(\Sigma)) .
$$

\section{A Basic Notions on Ultraproducts}

This appendix is much inspired by [15, chap. 2] with the notations of [2]. It is only provided for the convenience of casual readers. 
The set $\mathbb{N}$ is used as an index set on which we fix a (so called) non-principal ultrafilter $\mathscr{D}$. It is by definition a collection of infinite subsets of $\mathbb{N}$ closed under finite intersection, with the property that for any subset $E \subset \mathbb{N}$ either $E$ or its complement $\mathbb{N} \backslash E$ belongs to $\mathscr{D}$. In particular, the empty set does not belong to $\mathscr{D}$ and, if $E \in \mathscr{D}$ and $F$ is an arbitrary set containing $E$ then also $F \in \mathscr{D}$. Otherwise, $\mathbb{N} \backslash F \in \mathscr{D}$ and therefore, $\varnothing=E \cap(\mathbb{N} \backslash F) \in \mathscr{D}$ : a contradiction. Since every set in $\mathscr{D}$ must be infinite, it follows that every set whose complement is finite (such a set is called cofinite) belongs to $\mathscr{D}$.

Let $\mathscr{R}_{i}(i \in \mathbb{N})$ be a collection of rings. We form the ultraproduct $\mathscr{R}^{*}=$ $\left(\prod_{i \in \mathbb{N}} \mathscr{R}_{i}\right) / \mathscr{D}$ (or the ultrapower $\mathscr{R}^{\mathbb{N}} / \mathscr{D}$ if all rings $\mathscr{R}_{i}$ are the same ring $\mathscr{R}$ ) as follows. On the Cartesian product $\prod_{i \in \mathbb{N}} \mathscr{R}_{i}$ we define the equivalence relation: $a \equiv b$ if and only if the set of indices $i$ such that $a_{i}=b_{i}$ belongs to the ultrafilter $\mathscr{D}$.

We are going to use the following facts.

Consider an element $a \in \mathscr{R}^{*}$ which has no nonzero coordinates. The set of indices such that $a_{i}=0$ is empty. Since the empty set does not belong to the ultrafilter, $a \neq 0$ in $\mathscr{R}^{*}$.

Consider an element $a \in \mathscr{R}^{*}$ which has only finitely many nonzero coordinates. The set of indices such that $a_{i}=0$ is cofinite. Thus it belongs to $\mathscr{D}$. Thus $a=0$ in $\mathscr{R}^{*}$.

An ultraproduct of rings is a ring: addition and multiplication are performed componentwise. Let us prove that an ultraproduct of fields is a field (called an ultrafield). Consider some $a \neq 0$ in $\mathscr{R}^{*}$. Then the set $E=\left\{i \in \mathbb{N} \mid a_{i}=0\right\}$ does not belong to $\mathscr{D}$. Thus its complement $F=\mathbb{N} \backslash E$ belongs to $\mathscr{D}$. Define $\bar{a}$ as follows: for each $i \in \mathbb{N}$, if $a_{i}=0$ take $\bar{a}_{i}=0$ else take $\bar{a}_{i}=a_{i}^{-1}$. Let $u=a \bar{a}$. The set of indices such that $u_{i}=1$ is $F$, which belongs to $\mathscr{D}$. Thus $a$ admits an inverse and $\mathscr{R}^{*}$ is a field.

\section{Acknowledgements}

The authors would like to thank Zoé Chatzidakis and Mercedes Haiech for their help and their comments. The first author would like to acknowledge the support of the bilateral project ANR-17-CE40-0036 DFG-391322026 SYMBIONT. The second author was supported by the AustrianScience Fund (FWF): P 31327-N32.

\section{References}

1. Aroca, F., Garay, C., Toghani, Z.: The Fundamental Theorem of Tropical Differential Algebraic Geometry. Pacific J. Math. 283(2), 257-270 (2016)

2. Becker, J., Denef, J., Lipshitz, L., van den Dries, L.: Ultraproducts and Approximation in Local Rings I. Inventiones mathematicae 51, 189-203 (1979)

3. Boulier, F.: A Differential Algebra Introduction For Tropical Differential Geometry, Lecture Notes for the Workshop on Tropical Differential Geometry, Queen Mary College of the University of London, Dec. 2-7. Available at https://hal. archives-ouvertes.fr/hal-02378197 
4. Boulier, F., Haiech, M.: The Ritt-Raudenbush Theorem and Tropical Differential Geometry (2019), Preprint available at https://hal .archives-ouvertes.fr/ hal-02403365

5. Cotterill, E., Garay, C., Luviano, J.: Exploring tropical differential equations. Preprint available at http://arxiv.org/abs/2012.14067 (2020)

6. Denef, J., Lipshitz, L.: Power Series Solutions of Algebraic Differential Equations. Mathematische Annalen 267, 213-238 (1984)

7. Falkensteiner, S., Garay-López, C., Haiech, M., Noordman, M.P., Toghani, Z., Boulier, F.: The Fundamental Theorem of Tropical Partial Differential Algebraic Geometry. In: Proceedings of ISSAC 2020. pp. 178-185. Kalamata, Greece (2020)

8. Grigoriev, D.: Tropical Differential Equations. Advances in Applied Mathematics 82, 120-128 (2017)

9. Kaplansky, I.: Fields and Rings. The University of Chicago, Chicago, second edn. (1972)

10. Kolchin, E.R.: Differential Algebra and Algebraic Groups. Academic Press, New York (1973)

11. Lang, S.: Hilbert's Nullstellensatz in Infinite-Dimensional Space. Proceedings of the American Mathematical Society 3(3), 407-410 (1952)

12. Marker, D.: Model theory: an introduction, vol. 217. Springer Science \& Business Media (2006)

13. Ritt, J.F.: Differential equations from the algebraic standpoint, American Mathematical Society Colloquium Publications, vol. 14. American Mathematical Society, New York (1932)

14. Ritt, J.F.: Differential Algebra, American Mathematical Society Colloquium Publications, vol. 33. American Mathematical Society, New York (1950)

15. Schoutens, H.: The Use of Ultraproducts in Commutative Algebra. Springer Verlag (2010)

16. Singer, M.: The Model Theory of Ordered Differential Fields. Journal of Symbolic Logic 43(1), 82-91 (1978) 